\title{
Templated Synthesis of Desymmetrized [2]Catenanes With Excellent Translational Selectivity
}

\author{
Yi Liu*, Liana M. Klivansky, Saeed. I. Khan, and Xiyun Zhang
}

Contribution from the Molecular Foundry, Lawrence Berkeley National Laboratory, and

Department of Chemistry and Biochemistry, University of California, Los Angeles

\section{Supporting Information}




\section{Experimental Section}

General Methods: Reagents were purchased from Aldrich or synthesized as described. Thin-layer chromatography (TLC) was carried out using aluminum sheets, precoated with silica gel 60F (Merck 5554). The plates were inspected by UV-light. Melting points were determined on an Electrothermal MEL-TEMP 3.0 apparatus and are uncorrected. UV/Visible spectra were obtained using a Varian Cary 100 Bio spectrophotometer. Proton and carbon nuclear magnetic resonance spectra $\left({ }^{1} \mathrm{H}-\mathrm{NMR}\right.$ and

${ }^{13}$ C-NMR) spectra were recorded on a Bruker Avance500 II or DRX500, using the deuterated solvent as lock and the residual solvent as internal standard. All chemical shifts are quoted using the $\delta$ scale, and all coupling constants $(J)$ are expressed in Hertz $(\mathrm{Hz})$. Electrospray mass spectra (ESI-MS) were measured on a VG ProSpec triple focusing mass spectrometer. Matrix-assisted laser desorption ionization (MALDI) mass spectra were measured on 4800 MALDI TOF/TOF analyzer from Applied Biosystems.

Preparation of the PmI containing dibromide. As illustrated in Scheme S1, Pyromelletic anhydride reacts with two equiv. of 4-hydroxylbenzylamine (S2) at elevated temperature at $140{ }^{\circ} \mathrm{C}$ to give a diol $\mathbf{S 3}$. Bromination of $\mathbf{S 3}$ using $\mathrm{PBr}_{3}$ at $80{ }^{\circ} \mathrm{C}$ affords $\mathbf{3}$ as creamy solid.

Scheme S1. Preparation of the Pml-containing dibromide 3

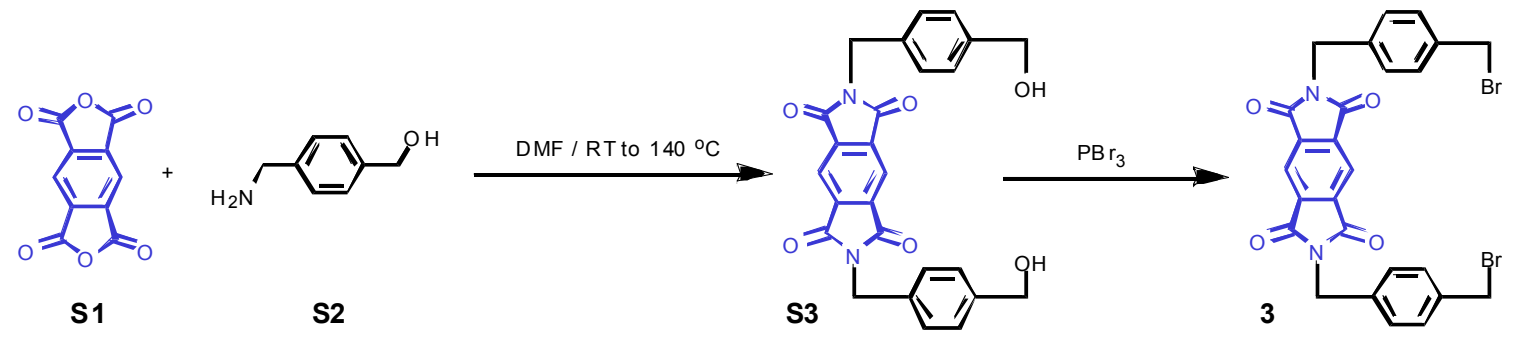


Synthesis of the diol S3. A mixture of pyromelletic anhydride (S1) $(1.07 \mathrm{~g}, 5.0 \mathrm{mmol})$ and 4-hydroxylbenzylamine (S2) (1.50 g, $11.0 \mathrm{mmol})$ was dissolved in DMF (20 mL) and stirred at room temperature for $2 \mathrm{hs}$. The reaction temperature was then raised and kept at $100{ }^{\circ} \mathrm{C}$ for $2 \mathrm{hs}$ and at $140{ }^{\circ} \mathrm{C}$ for another 30 mins. The resulting suspension was poured into water $(200 \mathrm{~mL})$, and the precipitate was collected by filtration and dried under vacuum to give $\mathbf{S 3}$ as cream-colored solid (2.15 g, 96\%). M.p. 324-326 ${ }^{\circ} \mathrm{C} ;{ }^{1} \mathrm{H}$ NMR $\left(\mathrm{CD}_{3} \mathrm{SOCD}_{3}, 500 \mathrm{MHz}, 298 \mathrm{~K}\right): \delta=8.23(\mathrm{~s}, 2 \mathrm{H}), 7.28(\mathrm{q}, J=8.0 \mathrm{~Hz}, 8 \mathrm{H}), 5.15(\mathrm{t}, J=$ $6.0 \mathrm{~Hz}, 2 \mathrm{H}), 4.80(\mathrm{~s}, 4 \mathrm{H}), 4.44(\mathrm{~d}, J=6.0 \mathrm{~Hz}, 4 \mathrm{H}) ;{ }^{13} \mathrm{C} \mathrm{NMR}\left(\mathrm{CD}_{3} \mathrm{SOCD}_{3}, 125 \mathrm{MHz}\right.$, $298 \mathrm{~K}): \delta=166.6,142.5,137.5,135.0,127.9,127.2,118.1,63.0,41.7$; MS (MALDITOF): $479.2(52 \%)[\mathrm{M}+\mathrm{Na}]^{+}$.

Synthesis of the dibromide 3. The diol $\mathbf{S 3}(0.50 \mathrm{~g}, 1.1 \mathrm{mmol})$ was suspended in DMF (20 $\mathrm{mL})$ and $\mathrm{PBr}_{3}(0.2 \mathrm{~mL})$ was added. The mixture was heated at $60{ }^{\circ} \mathrm{C}$ for $3 \mathrm{hs}$. The reaction mixture was quenched carefully with water, and the precipitate was collected by filtration and dried under vacuum to give 3 as cream-colored solid $(0.51 \mathrm{~g}, 80 \%)$. The compound was contaminated with small amount of impurity and was used without further purification. M.p. $>245{ }^{\circ} \mathrm{C}$ (dec.); ${ }^{1} \mathrm{H}$ NMR $\left(\mathrm{CD}_{3} \mathrm{SOCD}_{3}, 500 \mathrm{MHz}, 298 \mathrm{~K}\right): \delta=8.26$ (s, $2 \mathrm{H}), 7.42(\mathrm{~d}, J=8.0 \mathrm{~Hz}, 4 \mathrm{H}), 7.34(\mathrm{~d}, J=8.0 \mathrm{~Hz}, 4 \mathrm{H}), 4.83(\mathrm{~s}, 4 \mathrm{H}), 4.69(\mathrm{~s}, 4 \mathrm{H}) ;{ }^{13} \mathrm{C}$ NMR $\left(\mathrm{CD}_{3} \mathrm{SOCD}_{3}, 125 \mathrm{MHz}, 298 \mathrm{~K}\right): \delta=166.6,137.5,136.8,129.6,128.4,118.1,46.3$, 41.6; MS (MALDI-TOF): $501.1(10 \%)[\mathrm{M}-\mathrm{Br}]^{+}$. 
Synthesis of the HQ-based [2] catenane $5 \cdot 2 \mathrm{PF}_{6}$. A mixture of the dibromide 3 ( $0.10 \mathrm{~g}$, $0.17 \mathrm{mmol}), 4,4$ '-bipyridine (4) $(27 \mathrm{mg}, 0.17 \mathrm{mmol})$ and BPP34C10 (0.184 g, 0.34 mmol) in dry DMF $(10 \mathrm{~mL})$ was stirred at room temperature for 3 days. A red color was developed overnight. The resulting suspension was filtered and the filter disc was washed with Acetone. The combined organic solutions were evaporated and the residue was subjected to column chromatography $\left(\mathrm{SiO}_{2}, \mathrm{Me}_{2} \mathrm{CO}(100 \%)\right.$, then $\mathrm{NH}_{4} \mathrm{PF}_{6} / \mathrm{Me}_{2} \mathrm{CO}(0.2 \%$ $\mathrm{w} / \mathrm{v}))$. The red band was collected, evaporated and washed with water to give the product as red solid (72 mg, 30\%). M.p. $>250{ }^{\circ} \mathrm{C}$ (dec.); ${ }^{1} \mathrm{H}$ NMR $\left(\mathrm{CD}_{3} \mathrm{COCD}_{3}, 500 \mathrm{MHz}, 298\right.$ K): $\delta=9.30(\mathrm{~d}, J=6.5 \mathrm{~Hz}, 4 \mathrm{H}), 8.85(\mathrm{~d}, J=6.5 \mathrm{~Hz}, 4 \mathrm{H}), 7.89(\mathrm{~d}, J=8.0 \mathrm{~Hz}, 4 \mathrm{H}), 7.65$ (d, $J=8.0 \mathrm{~Hz}, 4 \mathrm{H}), 6.26(\mathrm{~s}, 4 \mathrm{H}), 5.95(\mathrm{~s}, 4 \mathrm{H}), 4.82(\mathrm{~s}, 4 \mathrm{H}), 4.02(\mathrm{~m}, 12 \mathrm{H}), 3.85(\mathrm{~m}, 12$ H), 3.70 (br s, 4 H), 3.56 (br s, 4 H), 3.47 (br s, 4 H); MS (ES+): 557.2 (100\%) [M$\left.2 \mathrm{PF}_{6}\right]^{2+}, 1259.3(2 \%)\left[M-\mathrm{PF}_{6}\right]^{+}$. HRMS (ESI) $\left[M-2 \mathrm{PF}_{6}\right]^{2+}$ : calcd 557.2288, found 557.2286 .

Synthesis of the DNP-based [2] catenane $6 \cdot 2 \mathrm{PF}_{6}$. A mixture of the dibromide $3(174 \mathrm{mg}$, $0.30 \mathrm{mmol}), 4,4$ '-bipyridine (4) (47 mg, $0.30 \mathrm{mmol})$ and 1/5DN38C10 (95 mg, 0.15 mmol) in dry DMF $(20 \mathrm{~mL})$ was stirred at room temperature for 10 days. A deep red color was developed during 3 hours. The resulting suspension was filtered and the filter disc was washed with Acetone. The combined organic solutions were evaporated and the residue was subjected to column chromatography $\left(\mathrm{SiO}_{2}, \mathrm{Me}_{2} \mathrm{CO}(100 \%)\right.$, then $\left.\mathrm{NH}_{4} \mathrm{PF}_{6} / \mathrm{Me}_{2} \mathrm{CO}(0.2 \% \mathrm{w} / \mathrm{v})\right)$. The purple band was collected and evaporated to give the product as purple solid (117 mg, 52\%). M.p. $>280{ }^{\circ} \mathrm{C}($ dec. $) ;{ }^{1} \mathrm{H}$ NMR $\left(\mathrm{CD}_{3} \mathrm{COCD}_{3}, 500\right.$ MHz, $298 \mathrm{~K}): \delta=9.12(\mathrm{~d}, J=6.5 \mathrm{~Hz}, 2 \mathrm{H}), 8.73(\mathrm{~d}, J=6.5 \mathrm{~Hz}, 2 \mathrm{H}), 8.14(\mathrm{~d}, J=8.0 \mathrm{~Hz}$, 
$2 \mathrm{H}), 7.92(\mathrm{~d}, J=8.0 \mathrm{~Hz}, 2 \mathrm{H}), 7.84(\mathrm{~d}, J=8.0 \mathrm{~Hz}, 2 \mathrm{H}), 7.68(\mathrm{~d}, J=8.0 \mathrm{~Hz}, 2 \mathrm{H}), 7.21$ (d, $J=8.5 \mathrm{~Hz}, 2 \mathrm{H}), 7.11$ (dd, $J=6.5 \mathrm{~Hz}, 2.5 \mathrm{~Hz}, 2 \mathrm{H}), 7.06$ (t, $J=8.0 \mathrm{~Hz}, 2 \mathrm{H}$ ), 6.98 (dd, $J=6.5 \mathrm{~Hz}, 2.5 \mathrm{~Hz}, 2 \mathrm{H}), 6.77$ (s, $2 \mathrm{H}), 6.40(\mathrm{~d}, J=7.5 \mathrm{~Hz}, 2 \mathrm{H}), 6.20(\mathrm{~d}, J=7.5 \mathrm{~Hz}, 2$ H), $6.10(\mathrm{~d}, J=13.5 \mathrm{~Hz}, 2 \mathrm{H}), 5.98(\mathrm{~d}, J=8.0 \mathrm{~Hz}, 2 \mathrm{H}), 5.92(\mathrm{~d}, J=13.5 \mathrm{~Hz}, 2 \mathrm{H}), 4.81$ (q, $J=13.5 \mathrm{~Hz}, 4 \mathrm{H}), 4.28(\mathrm{~m}, 4 \mathrm{H}), 4.11(\mathrm{~m}, 8 \mathrm{H}), 4.04(\mathrm{~m}, 6 \mathrm{H}), 3.90(\mathrm{~m}, 12 \mathrm{H}), 3.78$ (m, $2 \mathrm{H}), 2.95$ (d, $J=8.0 \mathrm{~Hz}, 2 \mathrm{H})$; MS (ES+): $607.2(100 \%)\left[M-2 \mathrm{PF}_{6}\right]^{2+}, 1359.5(3 \%)$ $\left[M-\mathrm{PF}_{6}\right]^{+}$. HRMS (ESI) $\left[M-2 \mathrm{PF}_{6}\right]^{2+}:$ calcd 607.2444 , found 607.2421.

$\mathrm{UV}-\mathrm{Vis}$ spectra of $[2]$ catenane $5 \cdot 2 \mathrm{PF}_{6}$ and $6 \cdot 2 \mathrm{PF}_{6}$

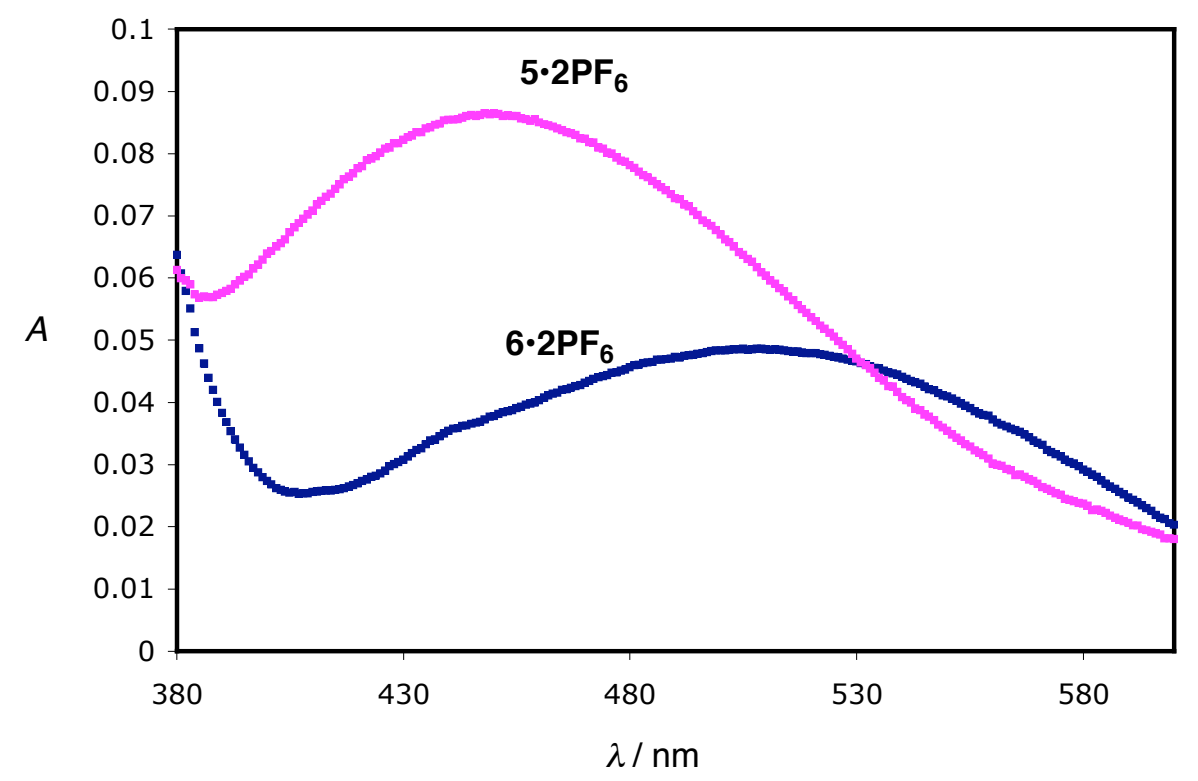

Figure S1. UV-vis spectra of $5 \cdot 2 \mathrm{PF}_{6}$ (in Acetone, $0.38 \mathrm{mM}$ ) and $\mathbf{6} \cdot 2 \mathrm{PF}_{6}$ (in Acetone, $0.11 \mathrm{mM}$ ) 


\section{${ }^{1} \mathrm{H}$ NMR Spectrum of Diol S3}
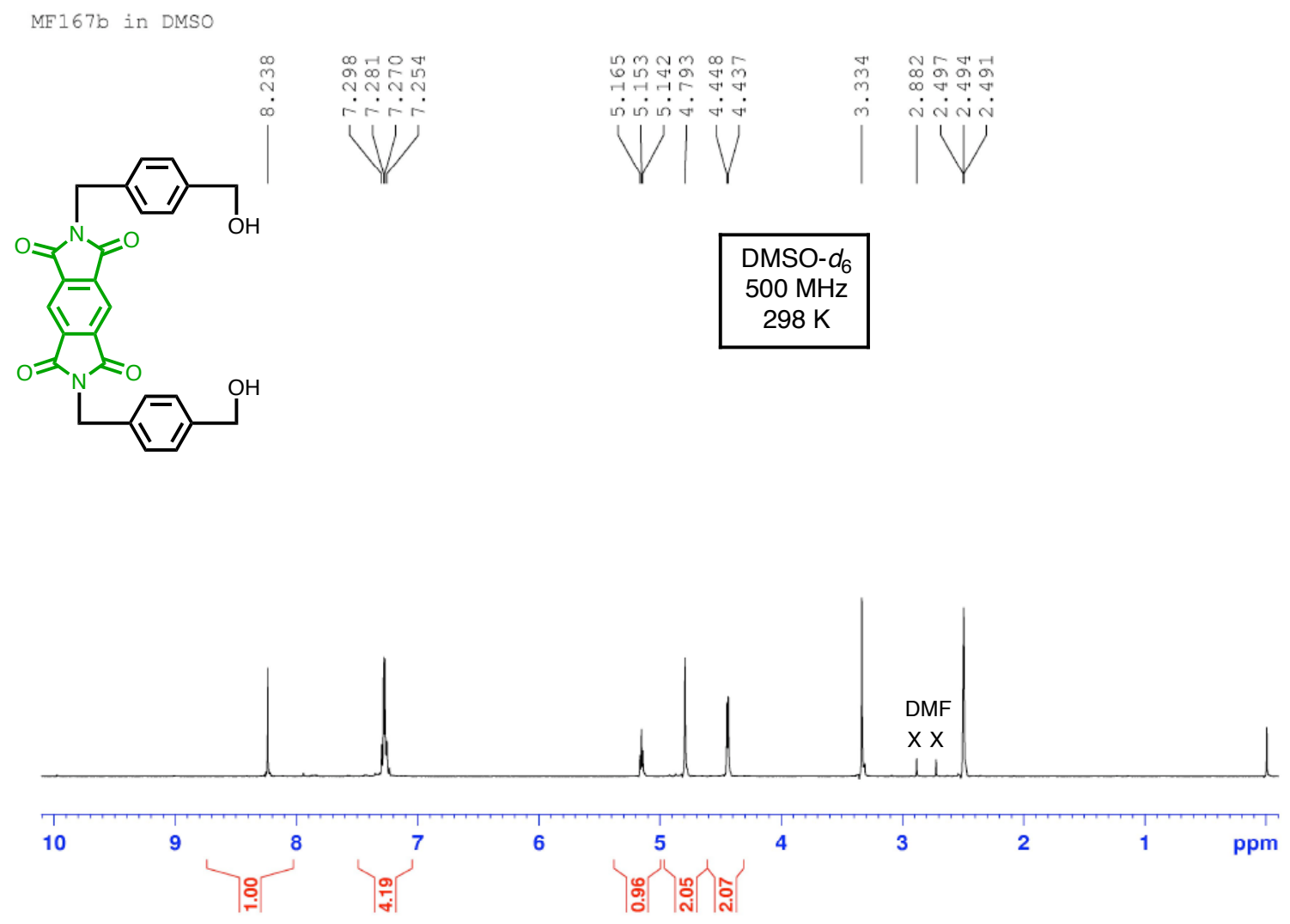


\section{${ }^{13} \mathrm{C}$ NMR Spectrum of Diol S3}

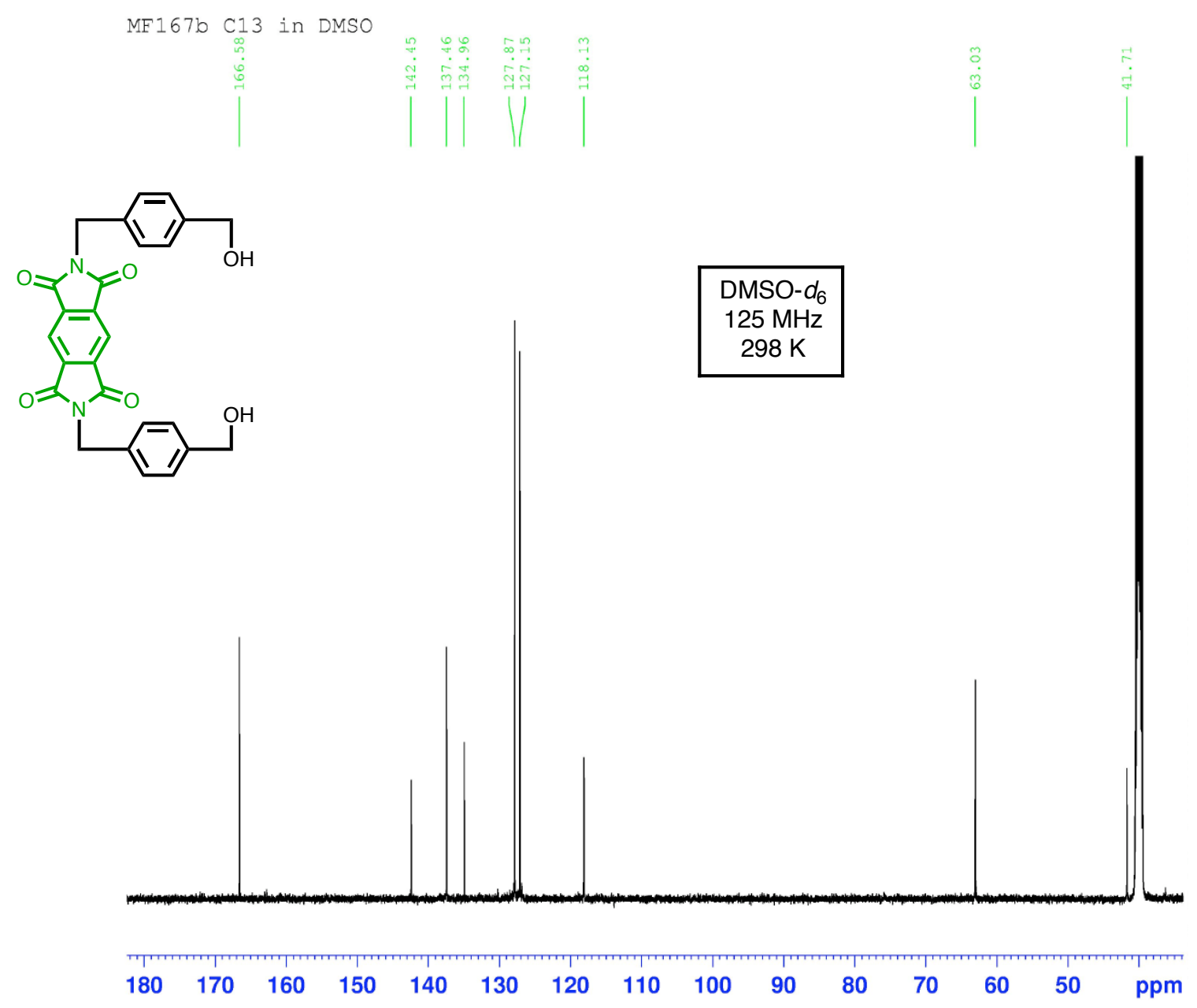


${ }^{1} \mathrm{H}$ NMR Spectrum of Dibromide 3

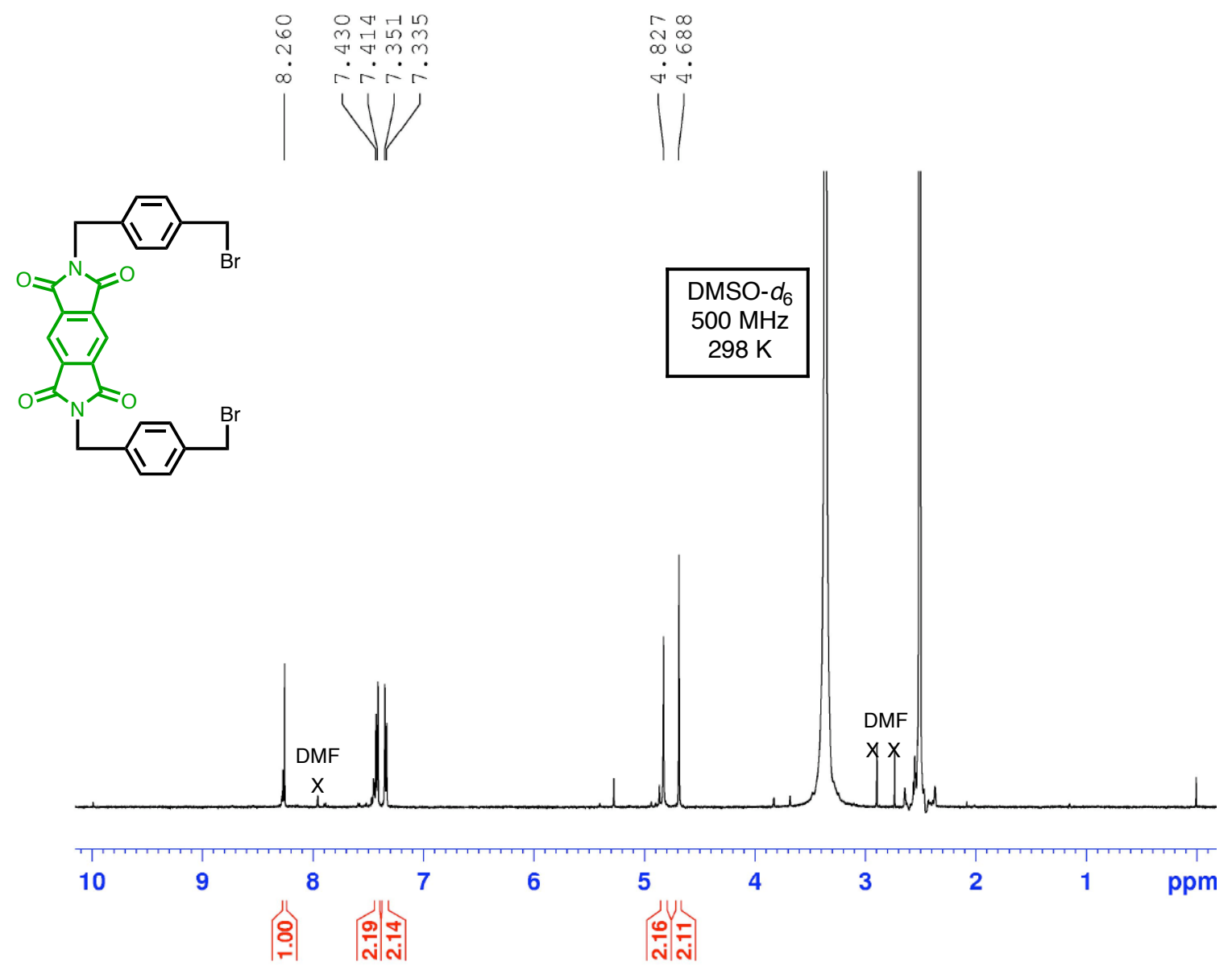




\section{${ }^{13} \mathrm{C}$ NMR Spectrum of Dibromide 3}
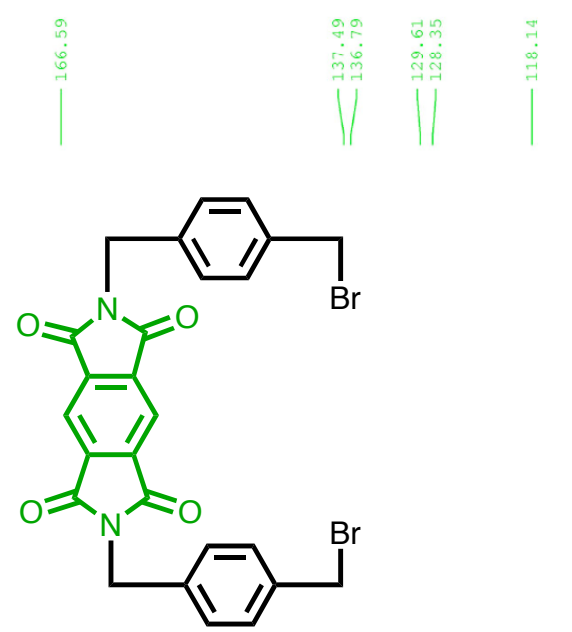
DMSO- $d_{6}$ $125 \mathrm{MHz}$ $298 \mathrm{~K}$

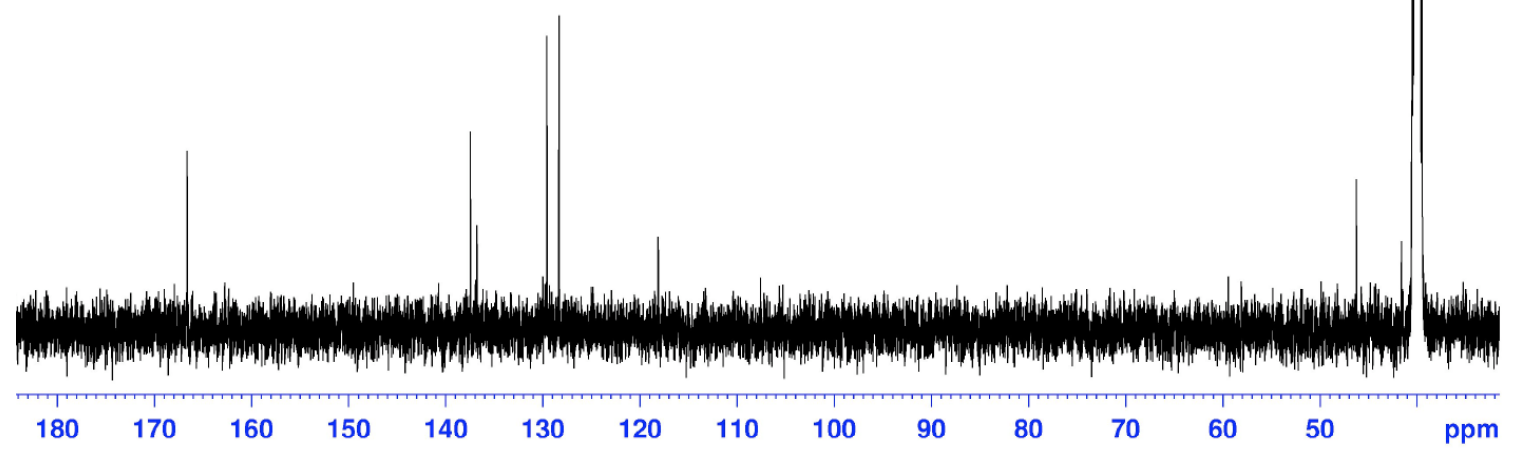


${ }^{1} \mathrm{H}$ NMR Spectrum of [2]Catenane $\mathbf{5} \cdot \mathbf{2} \mathbf{P F}_{6}$

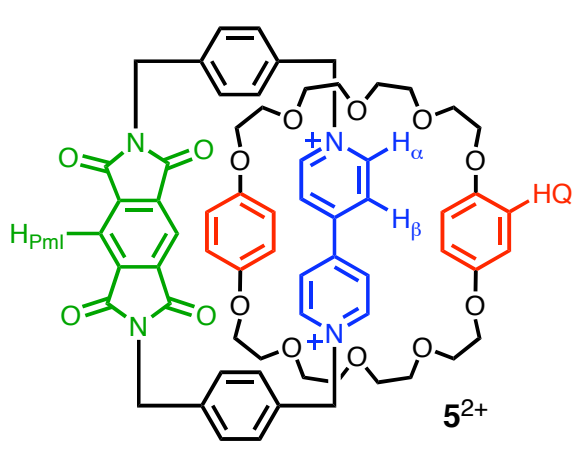

Acetone- $d_{6}$

$500 \mathrm{MHz}$ $298 \mathrm{~K}$

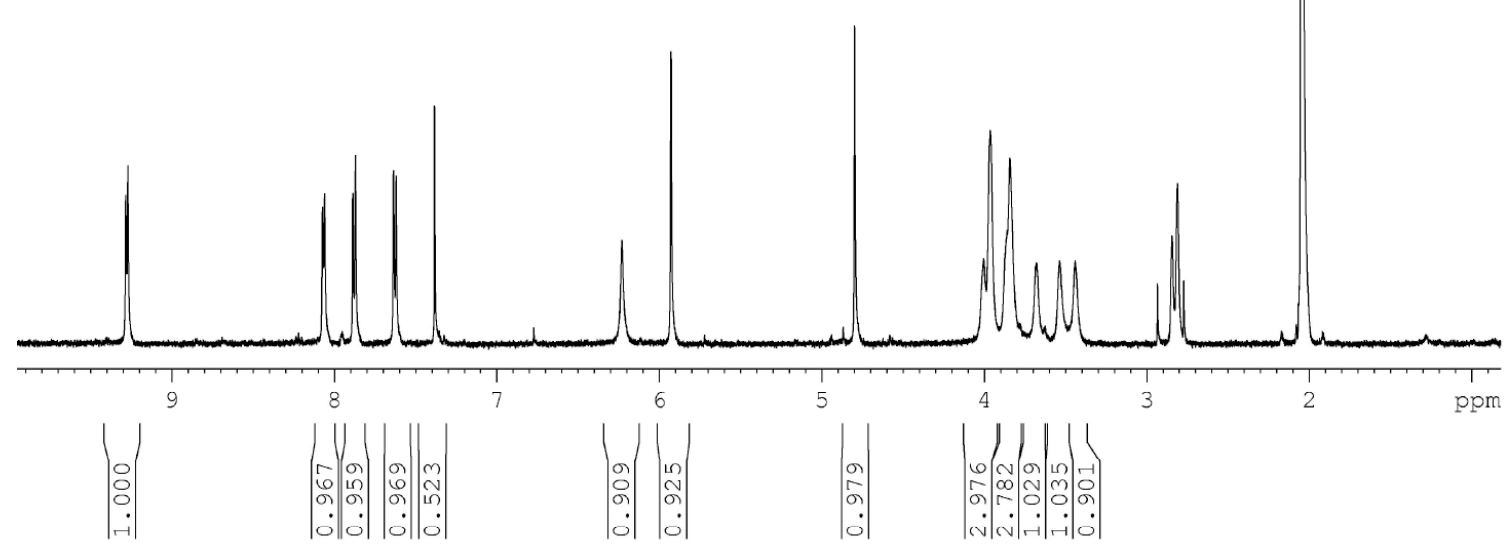


${ }^{1} \mathrm{H}$ NMR Spectrum of [2]Catenane $\mathbf{6} \cdot \mathbf{2 P F}_{6}$
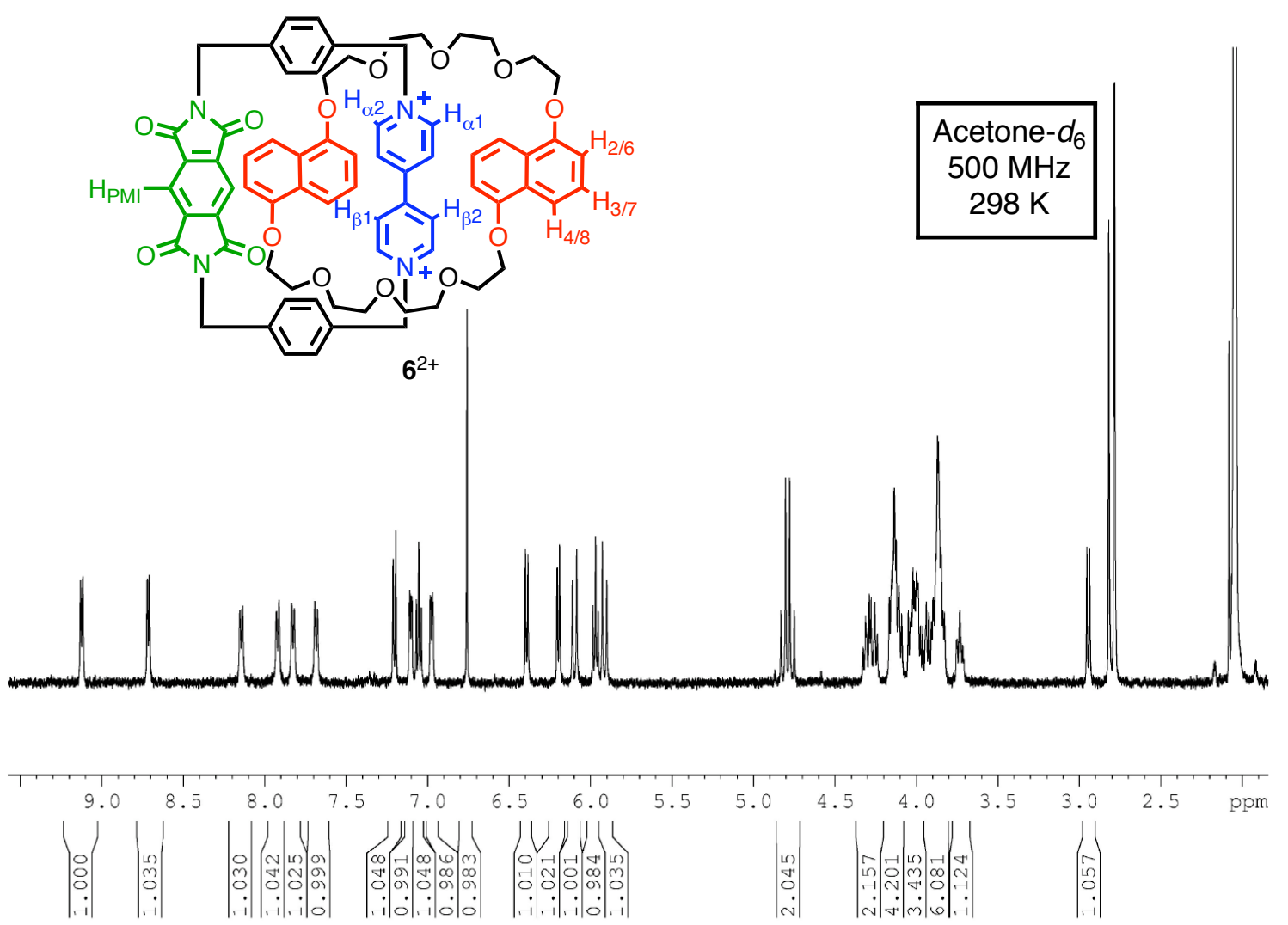